Cite this: Soft Matter, 2014, 10, 1365

\title{
Modeling crawling cell movement on soft engineered substrates $\uparrow$
}

\begin{abstract}
Jakob Löber, ${ }^{a}$ Falko Ziebert ${ }^{\text {bc }}$ and Igor S. Aranson ${ }^{\star d e}$
Self-propelled motion, emerging spontaneously or in response to external cues, is a hallmark of living organisms. Systems of self-propelled synthetic particles are also relevant for multiple applications, from targeted drug delivery to the design of self-healing materials. Self-propulsion relies on the force transfer to the surrounding. While self-propelled swimming in the bulk of liquids is fairly well characterized, many open questions remain in our understanding of self-propelled motion along substrates, such as in the case of crawling cells or related biomimetic objects. How is the force transfer organized and how does it interplay with the deformability of the moving object and the substrate? How do the spatially dependent traction distribution and adhesion dynamics give rise to complex cell behavior? How can we engineer a specific cell response on synthetic compliant substrates? Here we generalize our recently developed model for a crawling cell by incorporating locally resolved traction forces and substrate deformations. The model captures the generic structure of the traction force distribution and faithfully reproduces experimental observations, like the response of a cell on a gradient in substrate elasticity (durotaxis). It also exhibits complex modes of cell movement such as "bipedal" motion. Our work may guide experiments on cell traction force microscopy and substrate-based cell sorting and can be helpful for the design of biomimetic "crawlers" and active and reconfigurable self-healing materials.
\end{abstract}

Received 8th June 2013

Accepted 4th September 2013

DOI: $10.1039 / c 3 s m 51597 d$

www.rsc.org/softmatter on the skin of most fish and participating in its wound healing response. ${ }^{10-12}$ However, a thorough understanding of the functioning and design concepts of cellular substrate-based selfpropelled motion has not yet been fully achieved. It is therefore an important challenge to design and control synthetic selfpropelled substrate-based objects and to support these efforts by efficient modeling approaches..$^{13,14}$

In this work we generalize our recently developed model for a crawling cell ${ }^{15,16}$ to account for local traction force distributions and substrate deformations. In view of recent extensive studies of cells by traction force microscopy (see ref. 17 for a recent review), this step is crucial for a better understanding of force transfer mechanisms. We obtain traction and displacement patterns, quantities that so far have not been accessible to dynamic, self-consistent modeling. Furthermore we consider in detail the cell's dynamic modes of movement on homogeneous substrates. We find steady motion with fixed shape - as for keratocytes - but also stick-slip motion and various, more complex modes of movement. Especially, we have found a "bipedal"-like motion displaying periodic out-of-phase retractions of the trailing edges of the cell, similar to that recently found experimentally. ${ }^{18,19}$

The fact that motile cells have to navigate in complex environment (e.g. inside blood vessels or tissue) has inspired the studies of microswimmers moving through array of obstacles, ${ }^{20}$ for the purpose of sorting and separation, ${ }^{21}$ for rectification in ratchet-like channels or pumping fluid. ${ }^{22}$ This task is more 
complex for crawling cells. To emphasize the usefulness of our approach, we discuss cell motion on soft synthetic substrates, where the focus lies on substrates with engineered elastic properties. First, this design concept is more robust than the standard method of chemical surface patterning (where different densities of adhesion ligands are imprinted on the substrate's surface). Second, many studies revealed direct sensitivity of cells to the elasticity of the substrate, ${ }^{23,24}$ resulting in spectacular effects like e.g. durotaxis, ${ }^{25}$ i.e. the movement of cells in stiffness gradients. Our study demonstrated that cells moving on substrates with variable stiffness (characterized by the elastic modulus $G$ ) select a certain optimum stiffness value. Namely, cells on very soft substrates tend to move towards stiffer regions and oppositely on very stiff substrates. This finding, consistent with experimental observations, ${ }^{25,26}$ could be used for the design of engineered test assays for cell sorting, concentration, and separation.

\section{Brief description of the model}

Here we extend our recently developed model ${ }^{15,16}$ to account for local traction distribution and substrate deformations. We summarize only the basic modeling framework. Details on the model and the derivation of the spatially resolved substrate displacements can be found in Appendices A \& B.

Basic processes involved in substrate-based cell motility are protrusion via actin filament polymerization at the front of the cell (called the leading edge), intermittent formation of adhesion sites to transfer momentum to the substrate, and detachment of adhesion complexes and myosin motor-driven contraction at the cell's rear. ${ }^{27,28}$ In our model, all this complexity is cast into four continuous two-dimensional (2D) fields: the deformable and moving interface (the cell's membrane) is described by an auxiliary phase field ${ }^{29-32} \rho(x, y ; t)$ governed by an overdamped diffusive motion, $c f$. eqn (A.1), in a double-well model free energy. The latter has minima for the two "phases" [inside the cell $(\rho=1)$ and outside the cell $(\rho=0)$ ], whose levels determine the interface motion.

The propulsion mechanism, for most cells the ATP-consuming§ polymerization of actin filaments and the motor-induced actin network contraction, is modelled by a phenomenological eqn (A.2) for the vector field $\mathbf{p}(x, y ; t)$ describing the mean actin orientation, similar as done in the framework of polar liquid crystal theory developed for actin cytoskeleton bulk solutions. ${ }^{33-37}$

The coupling between these two fields is inspired by the underlying biological processes: ${ }^{15}$ actin is nucleated close to the membrane, $\uparrow$ with a rate $\beta$. On the other hand, existing actin that is polymerizing locally pushes against the membrane and

\$ Cells moving on a substrate often form a so-called lamellipodium, a thin layer of actin surrounded by the membrane, having a thickness of $\simeq 100 \mathrm{~nm}$. Compared to the typical length of actin filaments, several microns, one can hence assume a $2 \mathrm{D}$ situation.

$\S$ ATP $=$ adenosine triphosphate; ATP is delivered by the cell's metabolism and upon hydrolysis delivers an energy of $\simeq 20 k_{\mathrm{B}} T$ per molecule, which is used to drive both actin polymerization and molecular motors.

I By a cascade of initiators like WASP and Arp2/3. advects it, with a rate $\alpha$. Note that in contrast to a related study, ${ }^{\mathbf{3 8 , 3 9}}$ where the actin is treated by scalar fields, our approach offers direct access to the local actin network orientation, facilitating the implementation of traction forces. We emphasize that although the precise equations are obviously system specific, similar modeling approaches can be used in the context of synthetic systems. For example, self-propelled nanoparticle-laden microcapsules have been treated within a similar framework: ${ }^{14}$ there, instead of the vector field $\mathbf{p}$, a scalar field describing the concentration of nanoparticles contained in the deformable and permeable microcapsule induced the motion of the interface.

Explicit adhesion was implemented in ref. 16 by making the propulsion strength dependent on the number of formed adhesive bonds - linking the actin network to the substrate - by writing $\alpha \rightarrow \alpha(A) \simeq \alpha A$. Here $A(x, y ; t)$ is the density of adhesive bonds and for simplicity a linear dependence is assumed. Its dynamics is governed by a reaction-diffusion eqn (A.3), where bonds form with a certain rate (with the possibility of nonlinear effects). The bond detachment rate, in turn, is governed by the substrate deformation. Finally, the substrate is modeled as a $2 \mathrm{D}$ (height-averaged) viscoelastic medium, leading to the overdamped eqn (1) for the displacement field $\mathbf{u}(x, y ; t)$. The coupling to the cell is via the traction forces the latter exerts, as discussed below.

In our earlier study, ${ }^{\mathbf{1 6}}$ we modeled the effect of substrate elasticity by a single elastic spring, that described the elastic cellsubstrate interaction in a simplified coarse-grained fashion. However, locally resolved elastic displacements are needed to access the traction force and adhesion patterns inside the cell. Therefore, here we consider the spatially-resolved 2D elastic field coupled to the cell dynamics via the $2 \mathrm{D}$ traction distribution. This extension brings the model closer to reality in several ways. Firstly, it is known that the spatial distributions of traction and adhesive contacts are important for the directionality and guidance of the cellular motion, e.g. by stiffness gradients ${ }^{25}$ or adhesion patterns. ${ }^{\mathbf{4 0 , 4 1}}$ Secondly, locally-resolved displacements allow capturing relevant regimes of motion (e.g. bipedal motion ${ }^{18}$ ) that were absent in the previous approach. As in ref. 16, we consider a thin visco-elastic layer attached to a non-deformable surface. After integration across the layer's height, the inplane displacements $\mathbf{u}$ are of the form $(c f$. Appendix B)

$$
\partial_{t} \mathbf{u}=-\frac{1}{\eta}\left(G \mathbf{u}-\frac{1}{\xi}(\mathbf{T}+h[5 \Delta \mathbf{T}+19 \nabla(\nabla \cdot \mathbf{T})])\right),
$$

where $G=2 \tilde{G} / \xi H$ is the renormalized shear modulus of the layer, $\eta=2 \tilde{\eta} / \xi H$ describes viscous dissipation in the layer, $h=$ $H^{2} / 12$ is related to the thickness $H$ of the layer and $\mathbf{T}$ is the traction force exerted by the cell.

The coupling between the cell - whose shape is given by $\rho$ and that exerts local forces related to $\mathbf{p}$ - and the substrate, described by $\mathbf{u}$, is mediated by the adhesion bonds $A$. The coupling is provided by the traction distribution $\mathbf{T}$ exerted by the cell, which we model by assuming the following constitutive relation

$$
\mathbf{T}=-\xi A \rho \mathbf{p}+\xi A \rho \frac{\langle A \mathbf{p} \rho\rangle}{\langle A \rho\rangle} .
$$


Here the first term is due to actin polymerization, $\mathbf{T}_{\mathrm{pr}}=-\xi \rho A \mathbf{p}$, where $\xi$ is a coefficient characterizing the efficiency of force transmission and the "-" sign is present because the direction is opposite to the propulsion. The second term is the frictional part of the traction. Here we used the form $\mathbf{T}_{\mathrm{fr}}=\zeta \rho A$, i.e. it acts underneath the cell and is proportional to the number of adhesive bonds $A$. $\zeta$ is a (vector) quantity that has to be determined from the condition that the total traction is zero, as the cell does not exert a net force on the substrate. From this condition, i.e. $\langle\mathbf{T}\rangle=\left\langle\mathbf{T}_{\mathrm{pr}}+\mathbf{T}_{\mathrm{fr}}\right\rangle=0$, we obtain eqn (2). Note that the local $A, \rho$ and $\mathbf{p}$-values enter the traction force, $\|$ making the coupling to the substrate dependent on all other fields.

Eqn (A.1)-(A.3) are solved in a 2D periodic domain, which is done very efficiently by the quasi-spectral Fourier method implemented on GPUs (we typically used $512 \times 512$ FFT harmonics in double precision).

\section{Results}

\subsection{Traction force and substrate displacement patterns}

The forces exerted by cells on a substrate - either moving or spreading ones - can be obtained experimentally by the socalled traction force microscopy. In this method, fluorescent beads are immersed into a soft substrate and from their displacements during the action of cellular forces the traction forces can be calculated by solving an inverse problem. ${ }^{42,43}$ Alternatively, cells can be placed on microfabricated arrays of micro-pillars, ${ }^{44}$ their deflections directly giving the traction. Since its inception about 15 years ago, traction force microscopy has become a standard tool and has recently been automatized, ${ }^{45}$ as well as extended to $3 \mathrm{D}$ displacement fields. ${ }^{46}$ It has been applied to various cell types, e.g. to fibroblasts, ${ }^{47,48}$ keratocytes ${ }^{11}$ epithelial cells, ${ }^{49}$ neutrophils ${ }^{50}$ and glioma cells. ${ }^{51}$

Fig. 1 shows a typical modeling result for a steady moving cell (for the complex modes of motion see the next section). Panel (a) displays the phase field in color code and the actin orientation as arrows. Panel (b) shows the traction forces the cell locally exerts, and panel (c) depicts the resulting displacement field in the soft substrate (with the absolute values of the force and displacement color coded, respectively).

One sees from panel (b) that the traction force is maximum close to the motion-generating leading edge. This is a direct consequence of our implementation of the actin dynamics, eqn (A.2), that generates a pushing force (proportional to $\alpha$ ) at the front and accounts for contraction along $\mathbf{p}$ (proportional to $\sigma$ ). The displacement field is maximum in the bulk of the cell and its local orientation is similar to that of the traction pattern. The fact that the displacement is lacking behind is due to the interplay between the movement of the cell over the substrate and the viscoelastic relaxation of the adhesive layer.

We stress that our primary interest lies in the generic features of cell motility and cellular force transmission. Hence the obtained traction (and consequently the displacements) distribution is not necessarily applicable to a specific cell type.

|| Including a common factor $\rho$ is strictly speaking not necessary, but regularizes the numerical results.
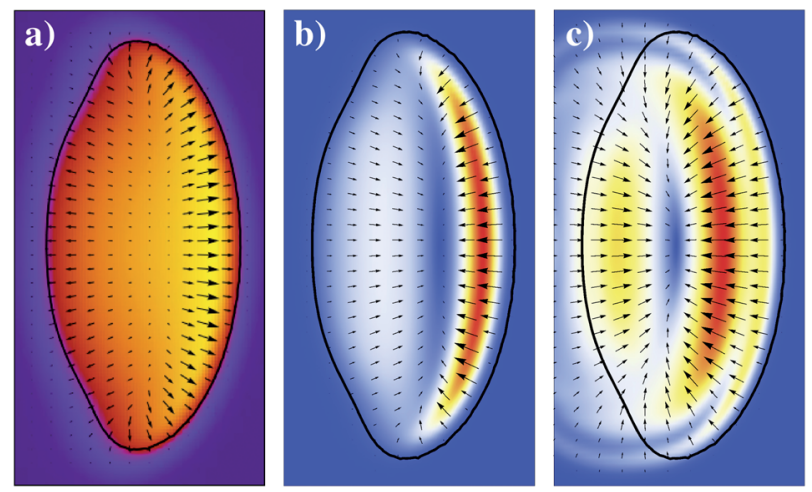

Fig. 1 (a) The cell shape (color coded; the black solid line shows the contour at $\rho=0.25$ ) and the internal actin orientation field $\mathrm{p}$ (shown by arrows). (b) The traction force T. Red (blue) corresponds to large (small) values of $|\mathrm{T}|$. (c) The displacement field $u$. Red (blue) corresponds to large (small) values of $|u|$. (a) - (c) are in the steady moving regime with parameters $G=0.25, \alpha=4, h=0.1$.

It is known (but not well understood) that different cell types exhibit different traction patterns - e.g. fibroblast cells have highest traction at the front, ${ }^{47}$ keratocytes at the sides ${ }^{11}$ and neutrophils at the rear..$^{50} \mathrm{At}$ the moment, as the model results in high traction at the front and a displacement field with similar direction as the traction, it perhaps best applies to fibroblasts, ${ }^{47,52}$ which however typically have different (and nonstationary) cell shapes. It also roughly applies to keratocytes: they have high traction at the front too. The fact that their highest traction is located at the sides is most probably a consequence of self-organized acto-myosin bundles spanning to the sides. ${ }^{11}$

Our generic model describes the coupling between the shape dynamics, actin, adhesion and traction distributions, and the resulting motion in a self-consistent fashion. It can be useful as a starting point for specific cell types. However, this task requires model ingredients that have to be extracted from dedicated experiments. For example, a faithful model of keratocytes needs an implementation of actomyosin bundles at the rear. On the other hand, to model fibroblasts the adhesion turnover kinetics has to be included. Since the turnover is much slower than that for keratocytes, it allows for partial maturation of the focal adhesions, ${ }^{24}$ which directly affects the actin dynamics (recruiting actin via a complex cascade), resulting in inhomogeneous adhesion and irregular motion. In addition, microtubules should be incorporated for fibroblasts as they constitute the stiffest part. ${ }^{53}$ Conceptually, it would be also interesting to modify the actin dynamics (eqn for $\mathbf{p}$ ), e.g. by including propulsion by treadmilling of actin filaments ${ }^{54}$ or viscoelastic actin-myosin flow inside the cell. ${ }^{55-57}$ Finally, the related question of how traction is self-organized internally and how it is related to the specific cell functions is obviously important, but beyond the scope of this study.

\subsection{Complex modes of movement}

Various types of complex cell movements have been reported: from contraction waves at the leading edge,$^{58}$ to stick-slip motion of parts of cells ${ }^{59}$ or whole cells, ${ }^{51}$ to bipedal motion. ${ }^{18,19}$ The latter 
are spontaneous shape oscillations during motion due to the coupling of the force-generation to the elasticity (of the substrate and of the cell), occur in keratocytes and result in lateral out-ofphase oscillations with respect to the direction of motion.

Fig. 2 shows a "phase diagram" of the motile states found in our model. We varied two crucial parameters: the substrate stiffness $G$ and the propulsion strength $\alpha$. We regain the generic motility modes that had been already obtained in: ${ }^{16}$ there are regions where no self-propelled motion is possible (blue circles), regions with steady moving or "gliding" states (green squares, see also ESI Movie $1 \dagger$ ) and an intermediate regime where stick-slip motion occurs (ESI Movie $2 \dagger$ ). Fig. 3 illustrates representative results for the stick-slip motion. Note that for amoebae, recently time-resolved traction forces could be related to alternating protrusion, contraction, retraction and relaxation cycles. ${ }^{\mathbf{6 0 , 6 1}}$ Although amoebae move differently (by pseudopods) and have a different adhesion mechanism (devoid of integrins), extensions of our modeling approach could be of value for this system as well.

In our work, ${ }^{16}$ the substrate was modeled as a single effective (visco-)elastic spring, resulting in simultaneous rupture of the adhesive bonds in the entire cell. This simplification, while useful, is not very realistic: it is known that some parts of cells still adhere while others lose contact. ${ }^{18,51,59}$ In other words, inhomogeneously distributed force affects the adhesion and provides a feedback on the overall shape and motion. This is remedied here, in contrast to ref. 16, by resolving the substrate displacement and traction locally. Due to the resulting local nature of the adhesive rupture, new dynamic states emerge at the boundaries of the transition from stick-slip to steady motion, $c f$. Fig. 2: (i) breathing motion (ESI Movie $3 \dagger$ ), where the cell extends periodically in the lateral direction (magenta down triangle). (ii) Bipedal motion, where the cell exhibits periodic lateral out-of-phase oscillations (ESI Movie $4 \dagger$ ) but keeps the initial propagation direction (orange up triangle) and (iii) wandering bipedal motion (ESI Movie $5 \dagger$ ), where the cell veers off the straight path and follows a slightly curved trajectory

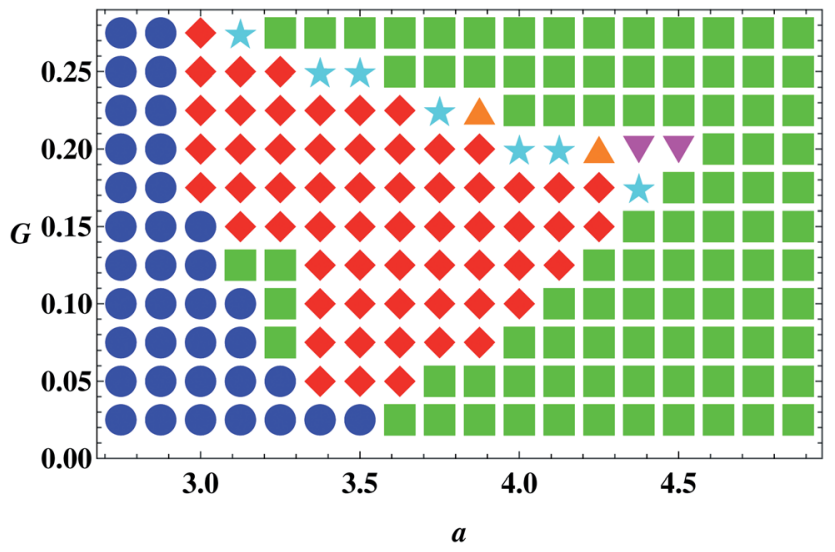

Fig. 2 Phase diagram for propulsion strength $\alpha$ vs. substrate's shear modulus G. denotes non-moving states, 1 steady moving (gliding) states, $\diamond$ stick-slip motion, $\star$ wandering bipedal and $\nabla, \Delta$ breathing and bipedal modes, respectively. Parameters are $U_{c}^{2}=0.2, \eta^{-1}=0.3, \beta=$ $\alpha / 2, h=0.1$. See also ESI Movies 1-5. $\dagger$
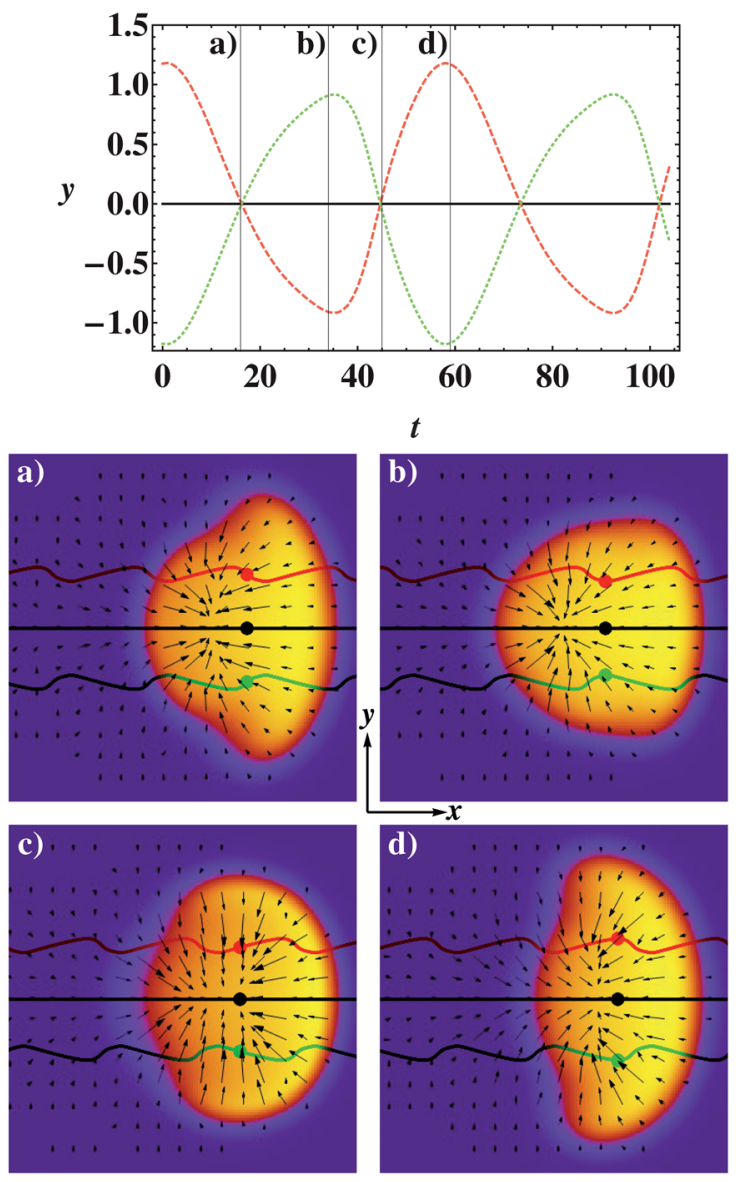

Fig. 3 Illustration of stick-slip motion. Two points out-of-center are marked to visualize the deformation. Panels (a)-(d) show the cell shape and the substrate displacement field. The upper diagram shows the $y$-components of the center of mass (c.o.m.) of the upper (dashed) and lower (dotted) half of the cell, as well as the overall c.o.m. position (black solid). The $x$-component does not show oscillations. Parameters are $G=0.125, \alpha=3.75$.

accompanied by asymmetric shape oscillations (light blue star). Some of these states stabilize after quite long transients of either gliding or stick-slip motion. Fig. 4 shows a representative bipedal motion, see also Appendix C for more details. Select trajectories and shapes for the wandering bipedal motion are shown in Fig. 5.

The instability leading to bipedal and more complex modes of movement is likely related to the coupling between various shape deformation modes and the translational mode. Somewhat similar behavior was recently observed in a model for selfpropelled "soft" deformable particles. ${ }^{62-64}$ There, the coupling between various intrinsic modes of shape deformations was implemented from symmetry arguments. In experiments, similar curved trajectories have been reported. ${ }^{18}$ The curved trajectories found in a certain parameter range of our model are interesting and should be investigated further. It should be noted, however, that even the very persistently moving keratocyte cells rarely take straight paths for more than several tens of their own length. On a larger scale, they rather explore their environment in a fashion resembling a random walk. 

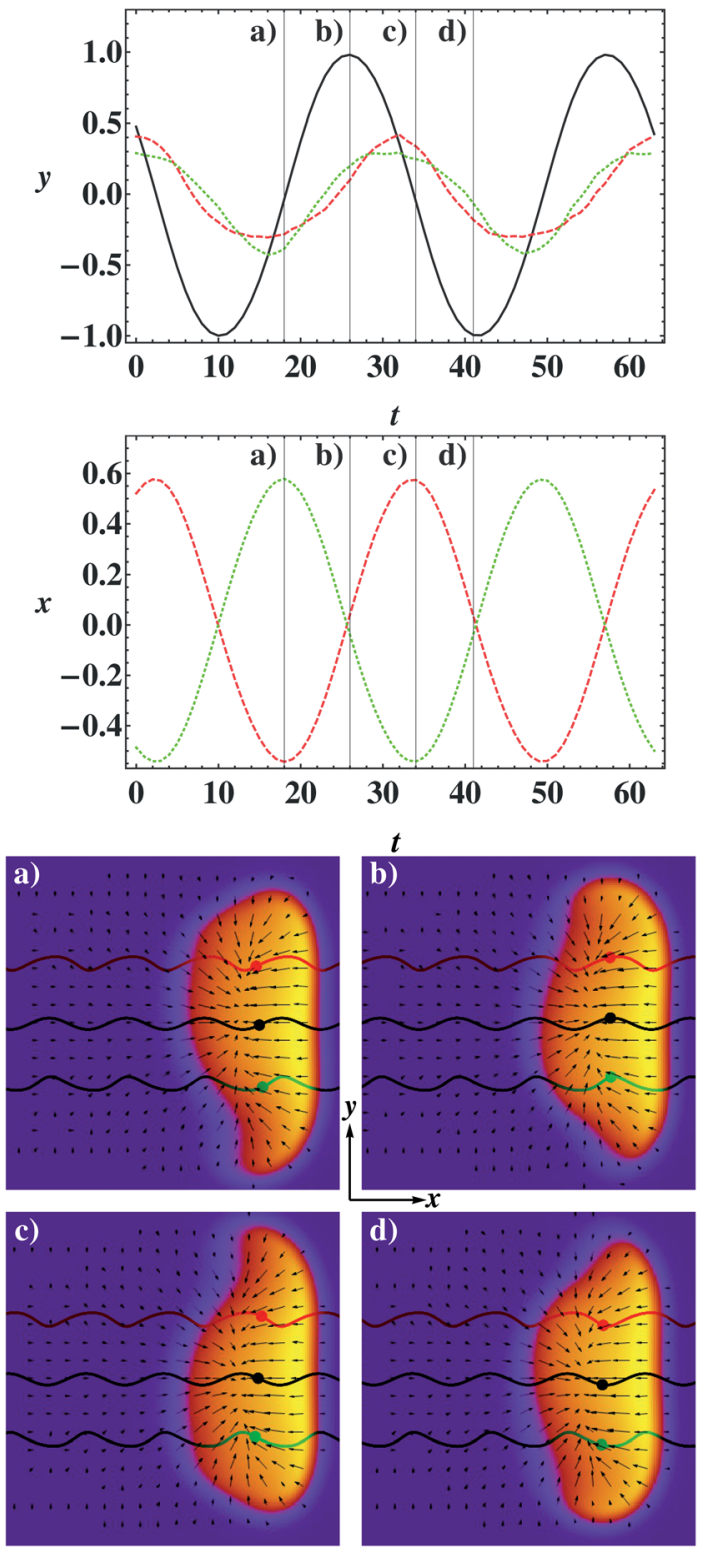

Fig. 4 Select results for the bipedal motion, i.e. an overall straight motion concomitant with out-of-phase oscillations of the lower and upper halves of the cell, see Appendix $C$ for details. Panels (a)-(d) show snapshots including the displacement field. The upper panel shows as dashed (dotted) the $y$-component of the c.o.m. of the upper (lower) halves of the cell which oscillate in-phase, and as black solid the $y$-coordinate of the c.o.m. of the entire cell. The central panel shows the out-of-phase oscillations of the corresponding $x$-coordinates. Parameters are $G=0.2$, $\alpha=4.25$. The green/red curves in the lowest panel are $r_{0}+r_{ \pm}$.

\subsection{Response to varying substrate elasticity}

In order to probe the response of the cell's motility machinery on a subcellular level, several groups have studied cells on chemically structured surfaces by patterning the surface with fibronectin, $\mathrm{RGD}^{\mathbf{1 2 , 4 0}}$ or other ligands for the adhesion receptor protein integrin, thereby modulating specific adhesion. In order to improve the mechanical stability and resistance to degradation, a possibly better design concept proposed recently are substrates with engineered elasticity. One possibility is the

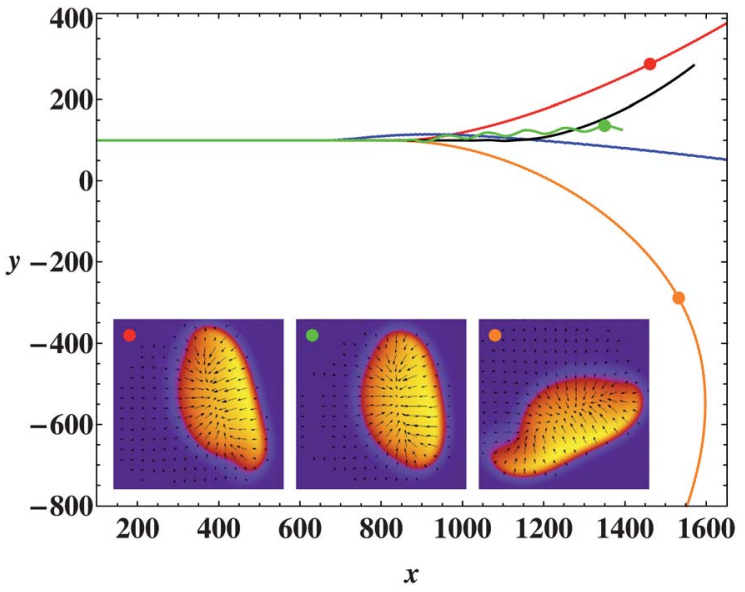

Fig. 5 Center of mass trajectories of wandering bipedal states. Parameters: $G=0.225, \alpha=3.75$ (red), $G=0.2, \alpha=4$ (blue), $G=0.25, \alpha=3.375$ (green), $G=0.25, \alpha=3.5$ (black) and $G=0.2, \alpha=4.125$ (orange). The inset shows representative cell shapes and displacement fields.

microfabrication of arrays of microposts (or pillars). Variation of their dimensions (height, thickness) results in local stiffness changes. ${ }^{26}$ However, pillars are not a very natural environment for cells, and new methods are currently under development to directly tune the modulus of a flat substrate, e.g. gradient materials ${ }^{65}$ and composite materials with alternating stiffness. ${ }^{66}$

We investigated the effects of a heterogeneous substrate shear modulus $G$, in order to obtain insights into the cell's mechanosensitivity and durotaxis. Note that the form of the equation for the substrate displacements, eqn (1), is unchanged in the thin layer limit $h \rightarrow 0$, cf. Appendix B. Within our modeling framework, we have found that cells prefer to stay (move) on a substrate of optimal stiffness: on very soft substrates, the cells migrate towards stiffer regions, while on very stiff substrates the cells move towards softer areas. This is exemplified in Fig. 6 for a linear gradient in $G$.

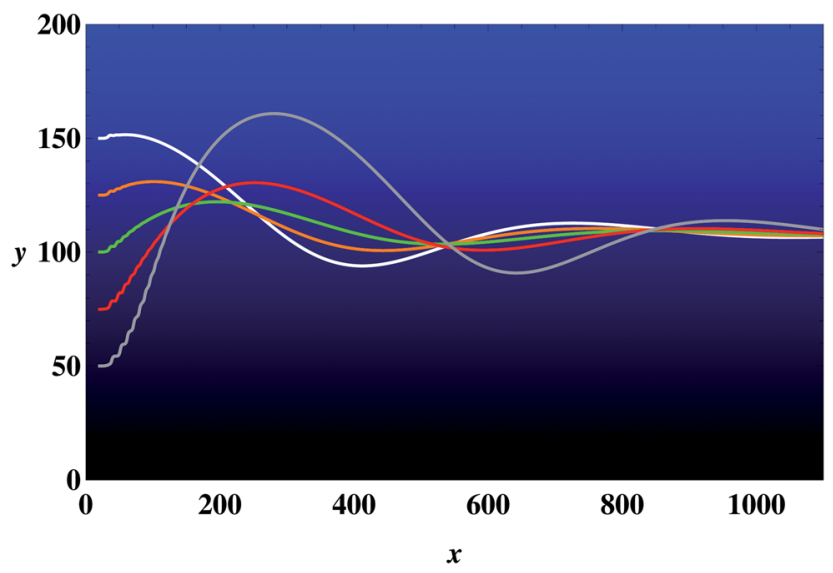

Fig. 6 Example of durotaxis: a linear gradient in the substrate's modulus $G$ is implemented in the $y$-direction, from $G=0$ (black) at the bottom to $G=0.4$ (blue) at the top. The curves show c.o.m. trajectories for different initial positions. They converge to an optimal value of $G$. Parameters: $\alpha=3.8, h \rightarrow 0$. 

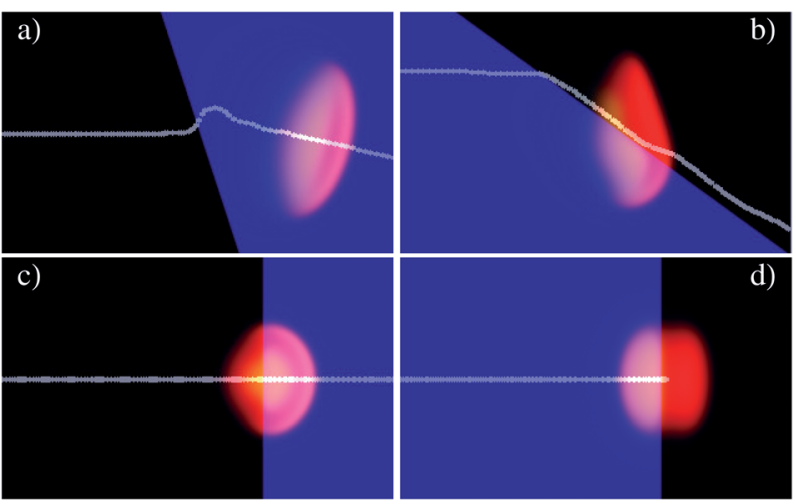

Fig. 7 Examples for the behavior of cells colliding with a step in the substrate stiffness (blue: $G=0.4$, black: $G=0.05$ ). The c.o.m. trajectories are shown in white. Top row: $\alpha=4=2 \beta$, bottom row: $\alpha=4, \beta=1.5$. Other parameters: $U_{\mathrm{c}}^{2}=0.25$.

We also observed a rich dynamics for cells encountering a step in substrate stiffness. Depending on the parameters (we studied mostly the propulsion strength $\alpha$, and varied the initial conditions), a variety of scenarios was observed, $c f$. Fig. 7. For cells moving at a certain angle towards a step from soft to stiff, we observe that the cell passes the step, $c f$. (a) and (c), including a possible deflection of the direction of motion, $c f$. (a). For cells moving from stiff to soft substrates, depending on the initial angle, the cell becomes either trapped and moves along the step, $c f$. panel (b), or is reflected from the step, $c f$. (d). This behavior is consistent with experimental observations of fibroblast cells on microposts assays. ${ }^{26}$

\section{Conclusions}

We developed a model for a crawling cell that accounts for the local traction forces exerted on the substrate and for the local substrate deformations. Insights from this study can be useful for other self-propelled objects on soft deformable substrates. Here we focused on the generic features of the emerging traction patterns, the possible motility modes and investigated the response of crawling cells on spatially varying mechanical properties of engineered substrates. Our analysis clearly shows that locally-resolved substrate deformations and traction forces are necessary for the description of nontrivial cell behavior, including mechanosensitivity, durotaxis, and complex modes of movement such as bipedal cell motion.

In earlier studies ${ }^{\mathbf{1 8 , 1 9}}$ the occurrence of bipedal motion was attributed to the intrinsic elasticity of the cell (modeled by internal elastic springs), rather than to the elasticity of substrate. In this context, one should note that the overall effect of substrate elasticity in many aspects is similar to the intrinsic elasticity of the cell. For example, deformations of both the cell and the substrate may promote breakage of adhesive bonds, resulting in an overall reduction of adhesion. This issue will be addressed in a future study, where cellular (visco-)elasticity will be added and their effects compared to those of the substrate elasticity.

Our modeling results indicated that, in a certain range of parameters, cells may exhibit erratic trajectories. While these types of motion are ubiquitous in experiments with moving cells, they are usually attributed either to the intrinsic randomness of cellular movements (stochastic formation of adhesive bonds, stochastic polymerization force, etc.) or to random heterogeneity of the substrate. Our work gives evidence that complex cell trajectories can also be an outcome of the intricate coupling between the various cell shape deformation modes via internal forces and the substrate.

Our modeling framework provides guidance for the interpretation of traction force microscopy data. "Prediction" of traction forces, however, requires model refinements towards cell type-specific internal actin and adhesion dynamics. The modeling provides a self-consistent description of cell movement on both homogeneous and heterogeneous substrates. Moreover, it suggests that substrates with engineered stiffness can be used as assays for cell sorting, separation, and concentration. The specific design (e.g. the jump in stiffness, and the angles, $c f$. Fig. 7) will be investigated in more detail in a future work, but will again be sensitive to the cell type-specific internal distribution of traction patterns.

Finally, the concepts developed in our work are general and can be applied to related, synthetic systems, e.g. to self-healing materials of nanoparticle-laden microcapsules moving on adhesive surfaces. We would also like to note that for such complex deformable and moving geometries as crawling cells or other soft self-propelled objects, the phase-field description presented here has significant advantages in terms of reducing the computational effort ${ }^{14}$ compared to direct simulations. ${ }^{13}$

\section{Appendix}

\section{A Phase-field model for a crawling cell}

The equations for the phase field and actin orientation read, for more details see, ${ }^{15,16}$

$$
\begin{gathered}
\partial_{t} \rho=D_{\rho} \Delta \rho-(1-\rho)(\delta-\rho) \rho-\alpha A \mathbf{p} \cdot(\nabla \rho) \\
\partial_{t} \mathbf{p}=D_{p} \Delta \mathbf{p}-\tau_{1}{ }^{-1} \mathbf{p}-\tau_{2}{ }^{-1}\left(1-\rho^{2}\right) \mathbf{p}-\beta f(\nabla \rho)-\gamma[(\nabla \rho) \cdot \mathbf{p}] \mathbf{p} .
\end{gathered}
$$

The parameter $\delta$ in eqn (A.1) controls the depth of the free energy minima and, therefore, governs the relative stability of the ${ }_{1} \rho=0,1$-phases. It was chosen to be $\delta=\frac{1}{2}+\mu\left(\langle\rho\rangle-A_{0}\right)-\sigma|\mathbf{p}|^{2}$, to ensure conservation of the cell's area $A_{0}=\pi r_{0}{ }^{2}\left(2^{\text {nd }}\right.$ term, $\langle.\rangle=.\int \mathrm{d} x \mathrm{~d} y$ ) and to allow for actin network contraction ( $3^{\text {rd }}$ term). The function $f(\kappa)=\frac{\kappa}{\sqrt{1+\varepsilon \kappa^{2}}}$ was introduced in eqn (A.2) to limit the creation of new actin filaments for too high $\kappa=\nabla \rho$.

The equation for the adhesion dynamics reads

$$
\partial_{t} A=D_{A} \Delta A+a_{0} \rho p^{2}+a_{\mathrm{n} 1} \rho A^{2}-s A^{3}-d(|\mathbf{u}|) A .
$$

It is coupled to the substrate displacement $\mathbf{u}$ via the detachment rate $d(|\mathbf{u}|)$. For simplicity we assumed a step-like function $d(|\mathbf{u}|)=0$ for $|\mathbf{u}|<U_{\mathrm{c}}$ and $d(|\mathbf{u}|)=d$ for $|\mathbf{u}|>U_{\mathrm{c}}$, which was implemented by taking $d(|\mathbf{u}|)=\frac{d}{2}\left(1+\tanh \left[b\left(\mathbf{u}^{2}-U_{\mathrm{c}}{ }^{2}\right)\right]\right)$ for large enough $b$. 


\section{B Derivation of the equation for the elastic displacements}

The stress tensor for an isotropic homogeneous incompressible visco-elastic solid (Kelvin-Voigt material) is given by ${ }^{67}$

$$
\sigma_{i k}=\tilde{G}\left(u_{i, k}+u_{k, i}\right)+\tilde{\eta}\left(\dot{u}_{i, k}+\dot{u}_{k, i}\right)-p \delta_{i k},
$$

where $u_{i}=u_{i}(x, y, z ; t), i \in\{x, y, z\}$ denotes the displacement field. $p=p(x, y, z ; t)$ is the pressure field and $\tilde{G}, \tilde{\eta}$ the shear modulus and viscosity. Within the assumption of overdamped motion $\left(\ddot{u}_{i}=0\right), \sigma_{i k, k}=0$ yields

$$
\begin{gathered}
\tilde{G} \nabla^{2} \mathbf{u}+\tilde{\eta} \nabla^{2} \dot{\mathbf{u}}=\nabla p, \\
\nabla \cdot \mathbf{u}=0 .
\end{gathered}
$$

We assume periodic boundary conditions in $x$ - and $y$-direction with period $L$ and vanishing displacements at the lower boundary of the substrate (non-deformable surface), $\mathbf{u}(x, y, z=$ $0, t)=0$. At the upper boundary, the cell exerts the traction force $\mathbf{T}$, but zero normal force on the substrate,

$$
\begin{gathered}
\sigma_{x z}(x, y, z=H, t)=T_{x}(x, y, t), \\
\sigma_{y z}(x, y, z=H, t)=T_{y}(x, y, t), \\
\sigma_{z z}(x, y, z=H, t)=0 .
\end{gathered}
$$

Nonlinearities arising from the free boundary at $z=H$ are neglected. Eqn (B.2) are equivalent to a biharmonic equation for $\mathbf{w}=\tilde{G} \mathbf{u}+\tilde{\eta} \dot{\mathbf{u}}$ and Laplace's equation for $p$. After Fourier transforming the $x$-and $y$-direction and introducing the wavenumber $k^{2}=k_{x}^{2}+k_{y}^{2}$, these equations become

$$
\begin{gathered}
\partial_{z}^{4} \mathbf{w}-2 k^{2} \partial_{z}{ }^{2} \mathbf{w}+k^{4} \mathbf{w}=0, \\
\partial_{z}{ }^{2} p-k^{2} p=0 .
\end{gathered}
$$

Six out of the necessary 14 boundary conditions for eqn (B.4) are given as before by $\mathbf{w}(x, y, z=0, t)=0$ and eqn (B.3), while the remaining eight boundary conditions are generated by evaluating eqn (B.2) at the boundaries. The assumption of a vertical substrate layer height $H$ much smaller than its horizontal extensions $L, H \ll L$, allows a long wavelength expansion $\left(k_{x}, k_{y}\right.$ $\ll 1 / H$ ) of the solution to eqn (B.4). We keep terms up to second order in $k_{x}, k_{y}$, which corresponds to retaining derivatives up to second order in space of the traction force T. Finally, integrating the result over $z$ from $z=0$ to $z=H$ leads to eqn (1).

Following from $\sigma_{i k, k}=0$, a heterogeneous substrate stiffness $\tilde{G}=\tilde{G}(x, y, z)$ gives a plethora of additional terms in eqn (B.2) involving all kinds of first order derivatives of $\tilde{G}$. However, we can neglect these terms if we truncate the long wavelength expansion at the lowest order and assume no dependence on the vertical direction, $\tilde{G}=\tilde{G}(x, y)$. Consequently, in eqn (1) we set $h \rightarrow 0$ and substitute $G \rightarrow G(x, y)$ for all computations involving a space-dependent substrate stiffness $G$.

\section{Characterization of the bipedal motion}

The overall center of mass (c.o.m.) is computed as [with $\mathbf{r}=$ $\left.(x, y)^{T}, \mathbf{r}_{0}=\left(x_{0}, y_{0}\right)^{T}\right]$

$$
\mathbf{r}_{0}(t)=\frac{1}{M_{0}} \int_{0}^{L} \int_{0}^{L} \mathrm{~d} x \mathrm{~d} y \mathbf{r} \rho(\mathbf{r}, t), M_{0}=\int_{0}^{L} \int_{0}^{L} \mathrm{~d} x \mathrm{~d} y \rho(\mathbf{r}, t) .
$$

Here $L$ is the size of the integration domain. During stick-slip and gliding motion, the cells have a symmetry axis passing through the center of mass and parallel to the direction of motion. Assuming that the direction of motion is always parallel to the $x$-direction (which is only true on average for the bipedal motion), we compute the c.o.m.'s of the upper $(+)$ and respectively lower $(-)$ half of the cell as

$$
\begin{gathered}
\mathbf{r}_{+}(t)=\frac{1}{M_{+}} \int_{0}^{L} \mathrm{~d} x \int_{y_{0}}^{L} \mathrm{~d} y\left[\mathbf{r}-\mathbf{r}_{0}(t)\right] \rho(\mathbf{r}, t), \\
\mathbf{r}_{-}(t)=\frac{1}{M_{-}} \int_{0}^{L} \mathrm{~d} x \int_{0}^{y_{0}} \mathrm{~d} y\left[\mathbf{r}-\mathbf{r}_{0}(t)\right] \rho(\mathbf{r}, t) \\
M_{+}=\int_{0}^{L} \mathrm{~d} x \int_{y_{0}}^{L} \mathrm{~d} y \rho(\mathbf{r}, t), M_{-}=\int_{0}^{L} \mathrm{~d} x \int_{0}^{y_{0}} \mathrm{~d} y \rho(\mathbf{r}, t) .
\end{gathered}
$$

\section{Parameters of the model}

The table displays the parameters of the model. If not mentioned otherwise, these values are used for all simulations.

\begin{tabular}{lll}
\hline Parameter & Value & Description \\
\hline$\alpha$ & $2-5$ & Propulsion rate \\
$\beta$ & $\alpha / 2$ & Actin nucleation rate \\
$\gamma$ & 0.5 & Motors' symmetry breaking \\
$\sigma$ & 1.3 & Motors' contraction \\
$\mu$ & 0.1 & Stiffness of volume conservation \\
$D_{\rho}$ & 1 & Stiffness of the diffuse interface \\
$D_{p}$ & 0.2 & Diffusion coefficient for $\mathbf{p}$ \\
$\tau_{1}{ }^{-1}$ & 0.1 & Degradation rate of actin \\
$\tau_{2}{ }^{-1}$ & 0.4 & Decay rate of p outside the cell \\
$\varepsilon$ & 37.25 & Regularization of actin creation \\
$D_{A}$ & 1 & Diffusion of adhesion sites \\
$a_{0}$ & 0.01 & Linear adhesion attachment rate \\
$a_{\mathrm{nl}}$ & 1.5 & Nonlinear adhesion attachment rate \\
$s$ & 1 & Saturation of adhesion sites \\
$d$ & 1 & Adhesion detachment rate \\
$b$ & 5 & Steepness of detachment transition \\
$U_{\mathrm{c}}{ }^{2}$ & 0.2 & Critical substrate stretch \\
$G$ & $0-0.5$ & Substrate modulus \\
$\eta$ & $10 / 3$ & Substrate dissipation \\
$h$ & 0.1 & Effective substrate layer thickness \\
$r_{0}$ & 15 & Radius of circular initial condition \\
& &
\end{tabular}

\section{Acknowledgements}

J. L. acknowledges financial support by the DFG via GRK 1558. F. Z. thanks the DFG for partial support via IRTG 1642 Soft Matter Science. The work of I.S.A. was supported by the U.S. Department of Energy, Office of Basic Energy Sciences, Division of Materials Science and Engineering, under Contract DE-AC02-06CH11357.

\section{References}

1 D. Bray, Cell movements: from molecules to motility, Garland Pub, 2001. 
2 W. F. Paxton, K. C. Kistler, C. C. Olmeda, A. Sen, S. K. S. Angelo, Y. Cao, T. E. Mallouk, P. E. Lammert and V. H. Crespi, J. Am. Chem. Soc., 2004, 126, 13424-13431.

3 W. Gao, S. Sattayasamitsathit, J. Orozco and J. Wang, J. Am. Chem. Soc., 2011, 133, 11862-11864.

4 A. Ghosh and P. Fischer, Nano Lett., 2009, 9, 2243-2245.

5 S. Sanchez, A. A. Solovev, S. M. Harazim and O. G. Schmidt, J. Am. Chem. Soc., 2011, 133, 701.

6 J. R. Howse, R. A. L. Jones, A. J. Ryan, T. Gough, R. Vafabakhsh and R. Golestanian, Phys. Rev. Lett., 2007, 99, 048102.

7 R. Dreyfus, J. Baudry, M. L. Roper, M. Fermigier, H. A. Stone and J. Bibette, Nature, 2005, 437, 862-865.

8 E. Lauga and T. R. Powers, Rep. Prog. Phys., 2009, 72, 09601. 9 I. S. Aranson, Phys.-Usp., 2013, 56, 79-92.

10 K. Keren, Z. Pincus, G. M. Allen, E. L. Barnhart, G. Marriott, A. Mogilner and J. A. Theriot, Nature, 2008, 453, 475.

11 M. F. Fournier, R. Sauser, D. Ambrosi, J.-J. Meister and A. B. Verkhovsky, J. Cell Biol., 2010, 188, 287.

12 E. L. Barnhart, K.-C. Lee, K. Keren, A. Mogilner and J. A. Theriot, PLoS Biol., 2011, 9, e1001059.

13 G. V. Kolmakov, R. Revanur, R. Tangirala, T. Emrick, T. P. Russell, A. J. Crosby and A. C. Balazs, ACS Nano, 2010, 4, 1115-1123.

14 G. V. Kolmakov, A. Schaefer, I. Aranson and A. C. Balazs, Soft Matter, 2012, 8, 180.

15 F. Ziebert, S. Swaminathan and I. S. Aranson, J. R. Soc., Interface, 2012, 9, 1084.

16 F. Ziebert and I. S. Aranson, PLoS One, 2013, 8, e64511.

17 J. H.-C. Wang and J.-S. Lin, Biomech. Model. Mechanobiol., 2007, 6, 361.

18 E. Barnhart, G. Allen, F. Jülicher and J. Theriot, Biophys. J., 2010, 98, 933-942.

19 A. J. Loosley and J. X. Tang, Phys. Rev. E: Stat., Nonlinear, Soft Matter Phys., 2012, 86, 031908.

20 G. Volpe, I. Buttinoni, D. Vogt, H.-J. Kümmerer and C. Bechinger, Soft Matter, 2011, 7, 8810.

21 W. Yang, V. R. Misko, K. Nelissen, M. Kong and F. M. Peeters, Soft Matter, 2012, 8, 5175.

22 P. K. Ghosh, V. R. Misko, F. Marchesoni and F. Nori, Phys. Rev. Lett., 2013, 110, 268301.

23 B. Ladoux and A. Nicolas, Rep. Prog. Phys., 2012, 75, 116601. 24 U. S. Schwarz and M. L. Gardel, J. Cell Sci., 2012, 125, 3051.

25 C. M. Lo, H. B. Wang, M. Dembo and Y. L. Wang, Biophys. J., 2000, 79, 144.

26 L. Trichet, J. Le Digabel, R. J. Hawkins, S. R. K. Vedula, M. Gupta, C. Ribrault, P. Hersen, R. Voituriez and B. Ladoux, Proc. Natl. Acad. Sci. U. S. A., 2012, 109, 6933.

27 M. Abercrombie, Proc. R. Soc. London, Ser. B, 1980, 207, 129. 28 M. Sheetz, D. Felsenfeld and C. Galbraith, Trends Cell Biol., 1998, 8, 51.

29 A. Karma and W.-J. Rappel, Phys. Rev. E: Stat. Phys., Plasmas, Fluids, Relat. Interdiscip. Top., 1998, 57, 4323.

30 I. S. Aranson, V. A. Kalatsky and V. M. Vinokur, Phys. Rev. Lett., 2000, 85, 118.

31 K. John, P. Peyla, K. Kassner, J. Prost and C. Misbah, Phys. Rev. Lett., 2008, 100, 068101.
32 W. Marth and A. Voigt, J. Math. Biol., 2013, 1-22.

33 K. Kruse, J. F. Joanny, F. Jülicher, J. Prost and K. Sekimoto, Eur. Phys. J. E, 2005, 16, 5.

34 H. R. Brand, H. Pleiner and F. Ziebert, Phys. Rev. E: Stat., Nonlinear, Soft Matter Phys., 2006, 74, 021713.

35 F. Ziebert, I. S. Aranson and L. S. Tsimring, New J. Phys., 2007, 9, 421.

36 C. Blanch-Mercader and J. Casademunt, Phys. Rev. Lett., 2013, 110, 078102.

37 E. Tjhung, D. Marenduzzo and M. E. Cates, Proc. Natl. Acad. Sci. U. S. A., 2012, 109, 12381-12386.

38 D. Shao, W. J. Rappel and H. Levine, Phys. Rev. Lett., 2010, 105, 108104.

39 D. Shao, H. Levine and W.-J. Rappel, Proc. Natl. Acad. Sci. U. S. A., 2012, 109, 6851.

40 G. Csucs, K. Quirin and G. Danuser, Cell Motil. Cytoskeleton, 2007, 64, 856-867.

41 M. Thery, V. Racine, M. Piel, A. Pepin, A. Dimitrov, Y. Chen, J. B. Sibarita and M. Bornens, Proc. Natl. Acad. Sci. U. S. A., 2006, 103, 19771.

42 M. Dembo and Y. L. Wang, Biophys. J., 1999, 76, 2307.

43 U. S. Schwarz, N. Q. Balaban, D. Riveline, A. Bershadsky, B. Geiger and S. A. Safran, Biophys. J., 2002, 83, 1380.

44 J. L. Tan, J. Tien, D. M. Pirone, D. S. Gray, K. Bhadriraju and C. S. Chen, Proc. Natl. Acad. Sci. U. S. A., 2003, 100, 1484 .

45 T. Das, T. K. Maiti and S. Chakraborty, Lab Chip, 2008, 8, 1308.

46 C. Franck, S. A. Maskarinec, D. A. Tirrell and G. Ravichandran, PLoS One, 2011, 6, e17833.

47 M. Dembo and Y. L. Wang, Biophys. J., 1999, 76, 2307.

48 S. Munevar, Y. L. Wang and M. Dembo, Biophys. J., 2001, 80, 1744.

49 M. L. Gardel, B. Sabass, L. Ji, G. Danuser, U. S. Schwarz and C. M. Waterman, J. Cell Biol., 2008, 183, 999.

50 L. A. Smith, H. Aranda-Espinoza, J. B. Haun, M. Dembo and D. A. Hammer, Biophys. J., 2007, 92, L58.

51 T. A. Ulrich, E. M. de Juan Pardo and S. Kumar, Cancer Res., 2009, 69, 4167.

52 D. Ambrosi, SIAM J. Appl. Math., 2006, 66, 2049.

53 A. Mogilner and K. Keren, Curr. Biol., 2009, 19, R762.

54 K. Doubrovinski and K. Kruse, Phys. Rev. Lett., 2011, 107, 258103.

55 B. Rubinstein, M. F. Fournier, K. Jacobson, A. B. Verkhovsky and A. Mogilner, Biophys. J., 2009, 97, 1853.

56 P. Recho and L. Truskinovsky, Phys. Rev. E: Stat., Nonlinear, Soft Matter Phys., 2013, 87, 022720.

57 A. Carlsson, New J. Phys., 2011, 13, 073009.

58 G. Giannone, B. J. Dubin-Thaler, H.-G. Döbereiner, N. Kieffer, A. R. Bresnick and M. P. Sheetz, Cell, 2004, 116, 431.

59 C. E. Chan and D. J. Odde, Science, 2008, 322, 1687.

60 R. Meili, B. Alonso-Latorre, J. C. del Alamo, R. A. Firtel and J. C. Lasheras, Mol. Biol. Cell, 2010, 21, 405.

61 D. Taniguchi, S. Ishihara, T. Oonuki, M. Honda-Kitahara, K. Kaneko and S. Sawai, Proc. Natl. Acad. Sci. U. S. A., 2013, 110, 5016-5021. 
62 T. Ohta and T. Ohkuma, Phys. Rev. Lett., 2009, 102, 154101.

63 M. Tarama and T. Ohta, Phys. Rev. E: Stat., Nonlinear, Soft Matter Phys., 2013, 87, 062912.

64 H. Kitahata, K. Iida and M. Nagayama, Phys. Rev. E: Stat., Nonlinear, Soft Matter Phys., 2013, 87, 010901.
65 K. U. Claussen, R. Giesa, T. Scheibel and H.-W. Schmidt, Macromol. Rapid Commun., 2012, 33, 206.

66 S.-Y. Chou, C.-M. Cheng and P. R. LeDuc, Biomaterials, 2009, 30, 3136.

67 L. Landau and E. Lifshitz, Theory of Elasticity: Vol. 7 of Course of Theoretical Physics, Elsevier, 1986. 